\title{
NADEZHdA KRUPSKAYA Y LAS MUJERES SOCIALISTAS EN LA REVOLUCIÓN RUSA, UNA MIRAdA FEMINISTA
}

\author{
NADEZHdA KRUPSKAYA AND THE SOCIALIST WOMEN OF \\ The Russian Revolution, A Feminist point of VieW
}

\section{Sara Lua González Forster*}

Resumen: El papel que algunas mujeres tuvieron en los momentos previos y posteriores a la Revolución rusa es, en muchas ocasiones, menospreciado o representado de manera puntual o anecdótica. Así, Nadezhda Krupskaya es descrita, en la mayoría de las publicaciones, tan solo como la esposa de Vladímir Ilich Uliánov -Lenin- a pesar de que tuvo un papel político muy importante en la antesala de la Revolución rusa de 1917, y posteriormente como parte del Comisariado de la Educación de la Unión Soviética, llamado Narkomprós. Este artículo se centra en la vida y en el trabajo político de Krupskaya, para mostrar a continuación algunos de los lazos que entabló con otras tres autoras socialistas de comienzos del siglo XX: Inessa Armand, Clara Zetkin y Alexandra Kollontai. A través de la vida y obra de Krupskaya, se pretende ampliar el panorama con la aportación de otras autoras socialistas de la época, y mostrar el vínculo que mantenía Krupskaya con su trabajo político, pensamiento y lucha por los derechos de las mujeres trabajadoras. Por último, se reflexiona en torno a la vigencia en la actualidad del pensamiento y las reivindicaciones de este grupo de mujeres socialistas.

Palabras clave: mujeres revolucionarias, feminismo y socialismo, Revolución rusa, derechos de las mujeres.

* Estudiante del Doctorado en Estudios Latinoamericanos en la Universidad Nacional Autónoma de México (UNAM); Maestra en Antropología Social por el Centro de Investigaciones y Estudios Superiores en Antropología Social (CIESAS); Licenciada en Antropología Social y Cultural por la Universidad Complutense de Madrid (UCM). Temas de investigación: Antropología de la educación, Movimientos sociales, Pedagogía Soviética en América Latina. Correo electrónico: saraluagonzalez@hotmail.com

Fecha de recepción: 2801 19; 2a. versión: 2603 19; Fecha de aceptación: 180619.

(cc) EY-NC-ND Páginas 43-72

julio-diciembre 2019, vol. 6

núm. 2 (13) ISSN: 2007-7610 
Abstract: The role that some women had prior and after the Russian Revolution has been in many cases underestimated or shown occasionally or in an anecdotal manner. Thereby, Nadezhda Krupskaya is described, in the majority of the journals, as only being the wife of Vladímir Ilich Uliánov -Lenin-, despite the fact that she had a very important political role in the prelude of the Russian Revolution of 1917, and afterwards as part of the Ministry of Education of the Soviet Union, called Narkomprós. This article follows the life and political work of Krupskaya, to show then some of the ties she established with three socialist authors at the start of the 20th century: Inessa Armand, Clara Zetkin and Alexandra Kollontai. Through the life and work of Krupskaya, it is our aim to widen the spectrum with the inputs of other socialist authors of the same period, and to show the link that Krupskaya kept with their political work, thought and struggle towards gaining working women's rights. Finally, we ponder about the relevance nowadays of the ideas and the demands of this group of socialist women.

Keywords: revolutionary women, feminism and socialism, Russian Revolution, women's rights.

\section{Introducción}

Estudiar a Nadezhda Krupskaya, 1869-1939, y el círculo personal y de trabajo político que entabló con otras mujeres socialistas, nos permite abrir una ventana necesaria para analizar esta efervescente época de debate, actividad y lucha por los derechos de las mujeres.

En el primer apartado de este artículo, se realiza una breve introducción a la relación entre el feminismo y el socialismo a finales del siglo XIX y principios del XX. Es importante señalar que las mujeres socialistas que presentamos en este escrito no se consideraban a sí mismas feministas, muy al contrario, asumían un fuerte distanciamiento con respecto al feminismo por considerarlo como parte de las demandas burguesas (Frencia y Gaido, 2018). Pero, como comprobaremos a lo largo del artículo, las acciones, ideas y contiendas políticas llevadas a cabo por estas mujeres socialistas hacen que desde nuestra óptica actual sea necesario incorporarlas como parte de la historia del feminismo.

En el segundo apartado, se presenta a la figura central del artículo, Nadezhda Krupskaya, mostrando aspectos biográficos de esta autora y la diversidad de 
actividades políticas, pedagógicas y en defensa de las mujeres que desarrolló a lo largo de su vida. El ámbito de escritura más fértil de Krupskaya era el educativo, pero también compuso textos en relación con las mujeres obreras y campesinas rusas y los problemas a los que tuvieron que enfrentarse antes y después de la Revolución rusa.

En el tercer, cuarto y quinto apartados, se exponen algunas de las ideas de la obra de Krupskaya en diálogo con las de otras tres autoras, Inessa Armand, Clara Zetkin y Alexandra Kollontai. Este artículo propone mostrar en forma de pares la relación de Krupskaya con cada una de estas tres grandes figuras del feminismo. Se describen las ideas de Krupskaya en torno a una tríada de ejes fundamentales, y en cada uno de ellos se vincula a Krupskaya con otra autora: con Inessa Armand, en relación con los derechos de las mujeres y de la infancia; con Clara Zetkin, en relación con la participación política de las mujeres; y con Alexandra Kollontai, en relación con la reestructuración de la familia.

En el sexto apartado se indica cómo las demandas y debates de estas mujeres socialistas mantienen una interlocución con las reivindicaciones de los potentes movimientos feministas actuales. Como caso paradigmático, se presentan las iniciativas que están llevando a cabo las mujeres del Sector de Género del Movimiento de los Trabajadores Rurales Sin Tierra-MST- en Brasil.

En las reflexiones finales, se delibera en torno a las aportaciones de las mujeres socialistas rusas en lo que se refiere a la relación entre el feminismo y el marxismo y cómo esos debates continúan en la actualidad. Se cierra el artículo resaltando los logros obtenidos durante este período por parte de las mujeres rusas y la importancia que tuvo la celebración del Día de la Mujer Trabajadora para la Revolución rusa.

\section{Feminismo y socialismo, una relación compleja}

Uno de los primeros intentos de poner en diálogo la situación de las mujeres con el socialismo surge desde la corriente de pensamiento del socialismo utópico en el siglo XIX: en Francia, en relación con el saintsimonianismo, y en Inglaterra, con el owenismo. Las mujeres de estos movimientos buscaron crear "proyectos alternativos de vida que cuestionaban las restricciones sociales impuestas sobre las mujeres” (Nash, 2004: 85) ${ }^{1}$.

\footnotetext{
${ }^{1}$ Algunas de estas acciones fueron, en el caso de las owenistas, el crear "sistemas de organización de la intendencia doméstica con cocinas y comedores colectivos y guarderías para las criaturas", 
Asimismo, el socialismo organizado en partidos políticos y el movimiento obrero del siglo XIX también se cuestionaron esta relación, pero enfatizaban que el problema de la situación de explotación de las mujeres residía en que las mujeres no tuvieran acceso al ámbito de la producción y, por ende, continuaran dependiendo de los hombres (De Miguel y Palomo, 2018). La solución estaría, por tanto, en la abolición de la propiedad privada y en el acceso de las mujeres al trabajo asalariado ${ }^{2}$. Consideraban que la emancipación de las mujeres "iba indisolublemente unida a la de la clase obrera, pues eran dos aspectos de una misma lucha: la que llevaba a la desaparición del sistema de producción capitalista” (Capel, 2008: 104). Augusto Bebel, con su obra La mujer y el socialismo de 1879 , es uno de los primeros en escribir sobre la especificidad de la situación de las mujeres dentro del socialismo ${ }^{3}$. "Bebel reconoce que la desigualdad podría deberse también a los hombres mismos, y no únicamente al sistema burgués. Pero subraya que la obtención de derechos puramente femeninos no resolverá el problema general de la alienación femenina y que únicamente la supresión del vínculo económico puede liberar a la mujer de la tutela masculina" (Navailh, 1993: 260). Esto se traducía en la idea de que las mujeres debían unirse a la lucha de clases del movimiento obrero y dentro de él podían crear organizaciones femeninas, pero sin llegar a establecer un feminismo obrero (Nash, 2004).

Como veremos más adelante, las mujeres que presentamos en este artículo plasmaron en sus escritos y en sus iniciativas políticas la importancia de hablar sobre las mujeres dentro del movimiento obrero. Sin embargo, eran críticas hacia el feminismo porque lo consideraban como una parte de la agenda política burguesa. Tanto Krupskaya como Kollontai tenían la certeza de que

al igual que exigir un "sistema de organización social basado en la cooperación mutua y la propiedad conjunta” (Nash, 2004: 86). En el caso de Francia, las mujeres seguidoras de SaintSimon se interesaron "por la realización de cambios sociales que mejorasen las condiciones del trabajo femenino y la estructura familiar" (ídem: 87). Además, crearon varias publicaciones, como la La Tribune des Femmes, que solo editaba textos escritos por mujeres.

${ }^{2}$ A pesar de estos planteamientos teóricos, dentro del movimiento obrero existía en la práctica una fuerte oposición de los hombres a que las mujeres comenzaran a tener trabajos asalariados (De Miguel y Palomo, 2018; Capel, 2008).

${ }^{3}$ Otra autora fundamental es la francesa-peruana Flora Tristán, que consiguió establecer la relación entre los problemas de las mujeres y la lucha de la clase obrera. "Sus escritos Peregrinación de una paria (1838) y Unión Obrera (1843) abrieron horizontes femeninos a los nuevos mundos de la modernización, de la industrialización y de la política del siglo XIX [...]. Flora Tristán fue una de las primeras mujeres en establecer una conexión ineludible entre la conquista del socialismo y el feminismo como vía de emancipación femenina” (Nash, 2004: 88). 
la lucha por los derechos de las mujeres se establecía a partir de la lucha de la clase proletaria. Por ejemplo, Kollontai, en el Primer Congreso de Mujeres de Toda Rusia, celebrado en 1908, en su texto "La mujer trabajadora en la sociedad contemporánea”, expone las diferencias entre el feminismo burgués -que en ese momento pedía el derecho a trabajar y tener acceso a los privilegios que los hombres de su clase poseían-, y las demandas de las mujeres obreras -que exigían mejores condiciones ya dentro del mundo laboral donde eran explotadas $^{-4}$.

A mediados del siglo XIX, la burguesa da sus primeros y tímidos pasos rumbo a la liberación financiera; ella toca insistentemente la puerta de universidades, talleres artísticos, oficinas. Y, mientras tanto, su "hermana pequeña", la proletaria, habiendo experimentado todo el horror de la explotación capitalista, de la fuerza de trabajo, exige al gobierno alguna intervención en el campo de la "libertad contractual" entre el capital y el trabajo (Kollontai, 2017: 151) .

La corriente de las mujeres socialistas en Rusia seguía de cerca al movimiento de las mujeres de Alemania, con Zetkin como líder y figura más representativa. Esta activista concentró grandes esfuerzos en conseguir derechos para la participación política de las mujeres pero, a pesar de propugnar el sufragio universal, no creía en la posibilidad de un feminismo obrero. Según Zetkin, "el feminismo y el marxismo no solo se diferencian por sus demandas y sus formas de organización, sino también por sus fundamentos teóricos: la teoría natural de las revoluciones burguesas, en un caso, y el materialismo histórico, en el otro" (Zetkin, 1907, en Frencia y Gaido, 2018: 7).

En el Tercer Congreso de la Internacional Socialista, celebrado en Zúrich en el año 1893, se produjo una "ruptura oficial de las socialistas con la ideología feminista, cuando Clara Zetkin introdujo la demanda de una legislación protectora para el trabajo femenino" (Frencia y Gaido, 2018:

\footnotetext{
${ }^{4}$ En relación con las divergencias entre el feminismo burgués y el feminismo de la clase trabajadora, en Estados Unidos, ya desde mediados del siglo XIX con el movimiento abolicionista se denunciaba la diferencia de clase y raza entre las mujeres. Mientras algunas mujeres reivindicaban el derecho a votar y a trabajar, otras, en este caso mujeres negras, habían sido esclavas y habían trabajado durante toda su vida. El discurso de Sojourner Truth en la Convención de las Mujeres en Ohio del año 1851, es un ejemplo de ello: "Miradme, ¡mirad mi brazo! He sembrado y plantado y llevado la cosecha al granero, y ningún hombre me podía adelantar. ¿Y no soy yo una mujer?” (Nash, 2004: 84).

${ }^{5}$ Cita traducida del portugués al español por la autora.
} 
19). Esta solicitud de protección para el trabajo de las mujeres propuesta por Zetkin aparece sintetizada al final del texto "La mujer trabajadora" ${ }^{\text {", escrito por }}$ Krupskaya en 1899 . Desde ese primer panfleto, Krupskaya ya realiza una clara crítica a la dependencia económica que tradicionalmente tenían las mujeres con respecto al padre o al marido. Señala la manera en que esa situación comenzaba a cambiar cuando las mujeres accedían al trabajo asalariado, pero cómo amargamente el menor salario de las mujeres en determinados trabajos desembocaba en "el florecimiento de la prostitución, abiertamente fomentada por los empleadores como un 'suplemento' al salario” (Frencia y Gaido, 2018: 18).

Krupskaya, Armand, Zetkin y Kollontai tienen un papel fundamental en la historia de las mujeres. Aun a pesar de la relación compleja que existía en esa época entre el feminismo y el socialismo, los debates que estas mujeres iniciaron han sido todavía objeto de reflexiones, estudios y reelaboraciones por parte del feminismo marxista (Sargent y Hartmann, 1981). Para adentrarnos en este terreno, el presente artículo propone centrarse en la vida y en las ideas de Krupskaya, y a través de ella conocer la lucha de las mujeres socialistas rusas por la educación, la participación política y los derechos como trabajadoras.

\section{Krupskaya: pedagoga, revolucionaria, bibliotecaria y escritora}

Lo sugestivo de centrar esta genealogía feminista en la figura de Krupskaya surge al darnos cuenta cómo una mujer bolchevique, figura fundamental en el Gobierno después de la Revolución rusa de 1917, sumamente creativa, pedagoga, traductora de textos y cartas y editora del periódico Iskra -entre otras muchas actividades que realizó a lo largo de su vida-, no era tan reconocida en el surgimiento y desarrollo de la Revolución rusa. Krupskaya, que además de ser militante escribió numerosos artículos y conferencias sobre la educación comunista, la juventud y la situación de las mujeres, quedó en muchos aspectos a la sombra de la figura de su esposo, Lenin, y de su obra, que ella se encargó de difundir después de su muerte. Sin embargo, su propio trabajo recibió mucha menos atención ${ }^{7}$.

\footnotetext{
${ }^{6}$ El artículo se publicó en 1900 firmado con el nombre de Sablina, pseudónimo de Nadezhda Krupskaya.

${ }^{7}$ Es necesario matizar en este punto que el desconocimiento de Krupskaya se refiere al ámbito internacional, ya que en Rusia es muy reconocida como pedagoga y existen diversos centros de investigación, de educación y premios académicos que llevan su nombre, al igual que un monumento en el centro de Moscú dedicado a su persona.
} 
Krupskaya nació en 1869 en San Petersburgo, en una familia que era de origen noble pero que había empobrecido. Su madre trabajaba como institutriz y su padre era militar. Debido a las ideas de su padre, que conocía el movimiento socialista, solían recibir visitas de algunos personajes de ideología revolucionaria, "al principio nihilistas, luego populistas y más tarde partidarios de la Sociedad Naródnaia Volia-Voluntad del pueblo-"8 (Krupskaya, 1978: 1). Desde joven, según relata ella misma en su autobiografía, ya manifestaba su aversión por los terratenientes que explotaban a los campesinos, además de firme oposición ante las injusticias y las guerras. También desde nińa, gracias a la amistad que entabló con una maestra rural a la cual acompañaba en sus labores, creció su interés por la educación y desde entonces quiso ser profesora. Su padre falleció cuando ella tenía 14 años y, al quedarse sola con su madre, tuvo que comenzar a trabajar desde esa temprana edad impartiendo clases en el liceo. Cuando era estudiante en la universidad, Krupskaya acudió a un círculo estudiantil marxista y, a partir de las lecturas de dicho grupo, llegó a la conclusión de que "el camino no era el autoperfeccionamiento tolstiano. La solución estaba en un poderoso movimiento obrero" (ídem: 5). Mientras estudiaba en la universidad, durante cinco ańos fue a la vez maestra en una escuela nocturna donde acudían obreros de diversas fábricas. Para ella, esa experiencia como docente fue fundamental: "Esos cinco años inocularon sangre viva en mi marxismo y me unieron para siempre con la clase obrera" (ídem: 6).

Durante ese tiempo, Krupskaya comenzó a formar una organización marxista y en 1894 entró en contacto con la Unión de Lucha para la Liberación de la Clase Obrera fundada por Lenin. En el año 1896, después de una huelga de tejedores, la policía realizó numerosas detenciones, entre ellas la de Krupskaya. Tras permanecer un tiempo en prisión, en 1898 es condenada al exilio, y en ese mismo año Lenin y Krupskaya contraen matrimonio en Siberia. Durante el destierro, escribe en diversos periódicos y forma parte de la Conferencia Internacional de las Mujeres Socialistas y entre 1905 y 1907 ejerce como secretaria del Comité Central del Partido.

\footnotetext{
${ }^{8}$ Naródnaia Volia -Voluntad del pueblo- fue una asociación creada en 1879 , que creía en la organización para la subversión y en la acción armada. Después de varios intentos, consiguieron acabar con la vida del emperador Alejandro II en 1881. Una de sus principales líderes fue una mujer, Sophia Perovskaya (Revista Nada, disponible en https://revistanada.com/2015/03/28/ narodnaya-volya-la-voluntad-del-pueblo/).
} 
La producción de esta autora es muy amplia, más de 3000 publicaciones entre los libros, panfletos, artículos y revistas que escribió (Skatkin y Cov'janov, 1994), sobre todo acerca de la educación comunista y la formación de la juventud. Ya desde antes de la Revolución de Octubre, algunas de las exigencias que Krupskaya demandaba a la que sería la futura escuela soviética -en un texto que escribió en el año 1913- eran que la educación debía ser universal, gratuita y obligatoria para todos. Remarcaba que debía incluir a ambos sexos, ser laica y con una organización democrática donde existiera una amplia participación de la población en la elección de los comités escolares, con plena libertad de opinión y con el derecho a recibir educación en su lengua nativa9 (Krupskaya, 2017: 31-34). Más adelante, Krupskaya consideraría importante romper esa división marcada entre el docente y la población e impulsar a que tanto los sindicatos como las cooperativas y organizaciones campesinas ayudasen a los maestros y maestras, se acercaran a la escuela e influyeran decisivamente en ella.

En cuanto a su labor política después de la Revolución de 1917, Krupskaya tuvo un papel fundamental en el Comisariado de Educación -Narkomprósy es considerada una de las fundadoras del sistema educativo soviético, especialmente en lo que respecta a las iniciativas de los primeros diez años. La hazaña educativa de la alfabetización de la población, cuyo departamento coordinaba Krupskaya, adquiere dimensiones colosales si consideramos el gran número de personas de todas las edades que pudieron tener acceso a la educación en un contexto de guerra civil, hambrunas, pobreza y masivo analfabetismo en la Rusia posterior a la Primera Guerra Mundial. "Al comenzar el siglo XX, casi el 80 por ciento de la población rusa entre 8 y 50 años era analfabeta" (Hernández Navarro, 2017: 13). En las primeras décadas de la existencia de la Unión Soviética, se consiguieron metas significativas en el ámbito educativo: "Fueron creados centros de educación superior por toda la Unión Soviética. Entre 1920 y 1940 fueron alfabetizados 60 millones de adultos. El 60 por ciento de la población no había cursado la educación básica en 1926, pero en 1930 el porcentaje ya había disminuido al 33 por ciento" (ídem: 13).

Pero, además de su labor como pedagoga en el Narkomprós, Krupskaya fue también una gran promotora de las bibliotecas, y aumentó los presupuestos

\footnotetext{
${ }^{9}$ Una de las iniciativas del Narkomprós fue crear libros escolares en las distintas lenguas de la Rusia Soviética; en 1928 se publicaron títulos en 70 lenguas nacionales, y para 1934 el número de idiomas en prensa ya alcanzaba los 104 (Skatkin y Cov'janov, 1994).
} 
que se dedicaban a sus instalaciones. Incluso publicó un artículo en el periódico Pravda donde argumentaba que la ampliación de las bibliotecas públicas era absolutamente necesaria, pero que, debido a la falta de presupuesto, la única solución era que la propiedad individual de los libros se tornara en propiedad colectiva. Poco después, todas las colecciones privadas de más de 500 ejemplares fueron confiscadas, nacionalizadas y guardadas en las nuevas bibliotecas (Haigh, 2007). No obstante, esta iniciativa también facilitó una censura, una criba de los libros, donde algunos títulos eran permitidos por considerarlos afines al socialismo, y otros pasaban a formar parte de "colecciones especiales” y no estaban disponibles para el público general (ibídem). Según Krupskaya, la alfabetización y el acceso de los libros para la población "permitían concienciar al pueblo sobre la revolución" (Muñoz-Muñoz, 2010), ya que estos eran considerados como instrumentos en la lucha socialista. También fue significativa la formación de bibliotecarios y bibliotecarias en los primeros años de la Revolución rusa. En 1918 se creó el primer seminario de la Biblioteca Soviética en Moscú por iniciativa de Krupskaya, y se abrieron más de 20 departamentos de bibliotecas en los Institutos Rusos de Educación Política, Institutos de Pedagogía y en las Academias de Educación Comunista (Haigh, 2007).

Después de la muerte de Lenin, en 1924, Krupskaya dedicó gran parte de su tiempo a editar la obra de su difunto esposo. En pleno auge del estalinismo, la figura de Krupskaya fue muy silenciada -se le otorgó el exclusivo papel de la viuda de Lenin-. Ella se mantuvo en la oposición política a Stalin, hasta que en 1927 decidió abandonar la oposición. Existen diversas teorías sobre esa decisión. Leon Trotsky (1939), en un texto que escribe con motivo del fallecimiento de Krupskaya ${ }^{10}$, señala que, en el convulso escenario de las desapariciones o asesinatos de gran parte del círculo cercano a Lenin y Krupskaya, dejar la oposición fue una estrategia para poder sobrevivir. Además, Trotsky remarca cómo cada paso de Krupskaya era vigilado, al igual que sus textos eran revisados y censurados. Otros autores atribuyen esa decisión a que, a pesar de ser contraria a las ideas de Stalin, Krupskaya era una persona muy leal al partido. En cualquier caso, se le otorgaron varias distinciones: la Bandera Roja del Trabajo en 1929, miembro honorario de la Academia de Ciencias de la URSS en 1931 y la Orden de Lenin en 1933. Falleció en 1939,

\footnotetext{
${ }^{10}$ El texto de Leon Trotsky, con el título "Krupskaya's Death", fue publicado en la revista The New International, con sede en Nueva York, en abril de 1939.
} 
a los 70 años de edad, y sus cenizas fueron depositadas junto a Lenin en su mausoleo; irónicamente, Stalin fue el que presidió su entierro.

Además de su fascinante vida como militante del partido y pedagoga dentro del Narkomprós, Krupskaya formó parte en la lucha por los derechos laborales de las mujeres, especialmente en los años anteriores a la Revolución de 1917. Algunos de los logros que se consiguieron para las mujeres en la nueva legislación soviética fueron "el derecho al voto y a ser candidatas, el derecho al divorcio, el acceso a la educación universal y gratuita, a un salario igual que el de los hombres, a ayudas para aliviar las cargas familiares y el derecho al aborto libre y gratuito" (Muñoz-Muñoz, 2010). Si tenemos en cuenta el contexto histórico, son logros sorprendentes y que constituyeron un gran avance en la lucha feminista. Un ejemplo de ello es que Rusia, en 1920, se convirtió en el primer país del mundo en legalizar el aborto a solicitud de la mujer. Además, se creó en 1919 un departamento en el gobierno, el Jenotdel, liderado primero por Armand y después por Kollontai. El Jenotdel trabajaba para mejorar las condiciones de vida de las mujeres en el ámbito educativo, en sus derechos en el matrimonio y en el trabajo. Lamentablemente, algunas de estas propuestas y medidas relevantes desde el punto de vista histórico para las mujeres rusas fueron frenadas al clausurarse el Jenotdel en 1930, sin encontrar apoyo de un partido comunista que en ese momento no focalizaba su interés en la lucha de las mujeres (Elwood, 1992). Posteriormente, en 1934 volvió a promulgarse una ley en contra de la homosexualidad en la Unión Soviética -cuando antes se había conseguido eliminar de la legislación el castigo a la homosexualidad- y en 1936 se restringió el aborto únicamente a aquellos casos en los que peligrara la salud de las madres. Los primeros años después de la Revolución rusa habían sido testigos de grandes logros en cuestiones como los derechos de las mujeres y la diversidad sexual, que más tarde fueron agresivamente eliminados: "Comprobamos una represión de la revolución sexual soviética; más todavía, una regresión a las formas autoritarias de la reglamentación moralizadora de la vida sexual" (Reich, 1985: 169).

Pero estos cambios a partir de los años 30 no empañan del todo los brillantes logros que se consiguieron por parte de Rusia en el período anterior. Es necesario destacar la importancia de las acciones de las mujeres que han sido totalmente invisibilizadas por las grandes figuras masculinas, propagando la falsa impresión de que fueron solo ellos los artífices de la Revolución rusa. Es llamativa la cantidad de artículos que mencionan el papel de relevantes 
mujeres rusas de la época como meras "ayudantes" de la Revolución que lideraron los hombres, o simplemente como sus amantes o esposas. El caso de Krupskaya es especialmente representativo de lo que ocurrió, porque tanto ella como su amiga Armand, que también participó en la Revolución de Octubre, son relegadas al papel de la esposa y la amante -respectivamente- de Lenin. Resulta indignante lo poco que se hace referencia a Armand como mujer revolucionaria y artífice fundamental del Jenotdel. Artículos de periódicos con títulos como "El revolucionario triángulo amoroso de Lenin" (Yegorov, 2017) o "Las tres mujeres que utilizó Lenin para el triunfo de la Revolución"11 (Felis, 2017) traducen desde la visión patriarcal la idea de que la vida amorosa de Lenin es más relevante que las acciones insurrectas de estas dos mujeres, al mostrarlas como "accesorios" de la lucha revolucionaria.

\section{La defensa de los derechos de las mujeres y la infancia: Krupskaya e Inessa Armand}

Inessa Armand, 1874-1920, aunque nació en Francia, a una temprana edad viaja a Rusia, donde es cuidada por su abuela y su tía. Desde 1903 forma parte del Partido Obrero Socialdemócrata Ruso y, debido a su trabajo de propaganda ilegal en Moscú, es condenada al exilio en el norte de Rusia, del cual escapa para llegar finalmente a París. En esta ciudad es donde entra en contacto con Lenin y con Krupskaya. Gracias a su dominio de los idiomas -Armand hablaba cinco lenguas-, es elegida para ser portavoz del Comité de Relaciones Exteriores (Elwood, 1992) y coordinar a los demás grupos bolcheviques que estaban surgiendo en Europa entre los años 1910 y 1917. En 1915, organiza en Suiza la Conferencia Internacional de Mujeres Socialistas y, al abdicar el zar Nicolás II, en abril de 1917 viaja de vuelta a Rusia, junto a Krupskaya, Lenin y otros compañeros, en un tren blindado. Después de la Revolución de Octubre, en 1919 se crea el Jenotdel con Armand como su dirigente principal, hasta el año 1920, cuando esta fallece a causa del cólera. Durante su vida, Armand luchó por la rehabilitación de las mujeres prostitutas, en la organización de las mujeres obreras y en la búsqueda de la equidad de las mujeres dentro del nuevo Estado Soviético (ibídem). Antes de fallecer, continúa con su labor como escritora y promueve la aparición del periódico feminista Kommunistka,

\footnotetext{
${ }^{11}$ En este artículo de Clara Felis del periódico El Mundo del 8 de marzo de 2017, "Las tres mujeres que utilizó Lenin para el triunfo de la Revolución” se refiere a Nadezhda Krupskaya, Inessa Armand y Alexandra Kollontai.
} 
que era considerado el portavoz del Jenotdel, y donde se escribía sobre la situación de las mujeres y sus derechos.

Armand fue una gran defensora de los logros de la Rusia Soviética en esos primeros tres años desde 1917 a 1920 y del papel que desempeñaron las mujeres dentro de la Revolución: "Con la Revolución de Octubre, tras el paso del poder a manos de los sóviets, la liberación completa de las obreras mediante la supresión de las viejas formas de la familia y la economía doméstica, no solo se ha vuelto posible, sino que es una de las condiciones necesarias de la instauración del socialismo" (Armand, 1920) ${ }^{12}$. Fue defensora de los derechos laborales de las mujeres durante el embarazo, del cambio del modelo de familia clásico hacia el de una unión libre. También se mostró profundamente crítica con la prostitución, que consideraba que debía ser totalmente erradicada y perseguidos los proxenetas que se beneficiaban con esta actividad. Para ella, la Revolución rusa significó liberarse de la opresión que surgía con el capitalismo, una triple explotación: en el Estado, en la fábrica y en la familia (ibídem).

Krupskaya conoció a Armand ya desde el exilio, y juntas escriben en el primer número del periódico Rabotnitsa -La obrera- ${ }^{13}$, en el cual también participaron otras renombradas mujeres bolcheviques como Ludmila Stahl y Alexandra Kollontai. La tirada de este periódico fue confiscada antes de que se pudiera publicar, pero finalmente, gracias a la labor de Anna UlianovaElizarova, que no había sido arrestada como lo fueron el resto de sus compañeras de edición, se pudieron imprimir doce mil ejemplares que fueron publicados con motivo del Día de la Mujer, en febrero de 1914 (Muñoz-Muñoz, 2010).

Tanto para Krupskaya como para Armand era muy importante la denuncia de la explotación de las mujeres trabajadoras, pero también la de los nińos y niñas que en muchas ocasiones trabajaban las mismas horas que los adultos. La infancia y la juventud eran asuntos primordiales para Krupskaya, que además creía en la posibilidad de la formación política de ambas. Durante el Gobierno soviético, se crearon grupos de la juventud comunista denominados Komsomoles. En un artículo de Krupskaya titulado "Importante sector del trabajo del Komsomol"14, la autora explica el trabajo realizado por parte de las jóvenes mujeres que pertenecían a estos grupos y cómo procuraban "llevar

\footnotetext{
${ }^{12}$ Firmado con el nombre Helène Blonina, pseudónimo de Inessa Armand.

${ }^{13}$ Aunque la primera edición del Rabotnitsa fue en 1914, al ser cerrada la revista por la represión zarista no se pudo reeditar hasta el año 1917.

${ }^{14}$ El artículo de Krupskaya apareció por primera vez en el número 8 de la revista Yuni kommunist -Joven comunista-, en el año 1935.
} 
Imagen 1. Revista Rabotnitsa, 1923. Se reinició en

1923 la edición siendo esta la portada del número 1 de la nueva etapa de la revista.

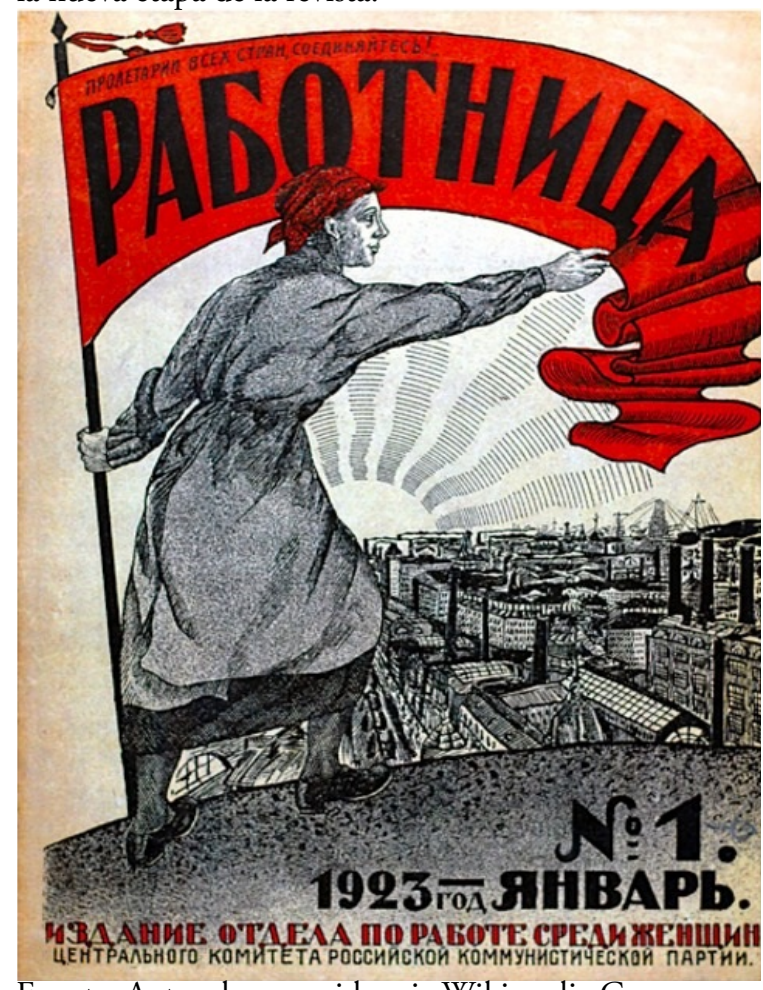

hasta el fin la emancipación de las mujeres" (Krupskaya, 1978: 48). Uno de los objetivos principales era conseguir que todas las jóvenes mujeres fueran a la escuela: "Hay que prestar especial atención a la defensa del derecho a estudiar y a la incorporación real de las muchachas al estudio" (ibídem).

Armand (1920) consideraba necesaria la instauración de una manutención pública para los niños -comida, ropa y educación-. Para Krupskaya, las largas horas de trabajo de las mujeres en la fábrica tenían como principal consecuencia que muchos de los niños y niñas creciesen en las calles, y en algunos casos, las madres, sin capacidad ni tiempo para educarlos, decidían internar a sus hijos en orfanatos. Para estas dos autoras, la solución se hallaba tanto en acabar con la explotación de las mujeres trabajadoras como en que la sociedad garantizase la educación y la alimentación para la infancia. Propusieron la creación de jardines de infancia que permitirían a las mujeres ir a trabajar con la certeza 
de que sus hijos estarían bien cuidados y alimentados. Además, la educación que los niños y niñas recibirían en la escuela pública tomaría como base la importancia del trabajo productivo, creativo y multilateral, con el fin de:

Formar personas desarrolladas multilateralmente, con predisposiciones sociales conscientes y organizadas, que tengan una visión del mundo reflexiva, integral y que claramente entiendan todo lo que está aconteciendo a su alrededor en la naturaleza y en la vida social; personas preparadas en la teoría y en la práctica para todos los tipos de trabajo, tanto físico como mental; personas capaces de construir una vida social racional, llena de contenido, bonita y alegre. Esas personas son necesarias para la sociedad socialista, sin ellas el socialismo no se puede realizar plenamente (Krupskaya, 2017: 70) ${ }^{15}$.

\section{La participación política de las mujeres: Krupskaya y Clara Zetkin}

Además de sus lazos con Armand, Krupskaya también formó parte de otras iniciativas de las activistas socialistas en su defensa por los derechos laborales y la participación política de las mujeres. En 1915 acude como parte de la delegación rusa a la Tercera Conferencia Internacional de Mujeres Socialistas, en la cual, junto a Zetkin, fue la Secretaria del Buró Socialista Femenino Internacional (Muñoz-Muñoz, 2010).

Clara Zetkin, 1857-1933, fue una de las mujeres más importantes dentro del movimiento feminista alemán de principios del siglo $\mathrm{XX}$, con una trayectoria extensa como militante socialista, al igual que en la defensa de los derechos de las mujeres. En todos sus escritos, la participación política de las mujeres dentro del partido comunista constituyó una de sus consignas más poderosas, al igual que un profundo antimperialismo y antimilitarismo. No participó en la Revolución rusa como las demás autoras que presentamos, pero tuvo una importante influencia en la lucha por los derechos de las mujeres de esa época y en la propia obra de Krupskaya.

Zetkin era una militante socialista muy activa, formó parte del grupo "Internacional", fue una de las creadoras de la revista Die Internationale en 1915 y, más adelante, en 1918, fue una de las fundadoras del Partido Comunista Alemán. Posteriormente, fue nombrada secretaria general del Secretariado Internacional de Mujeres y también miembro del Comité Ejecutivo de la

${ }^{15}$ Fragmento del texto "Sobre la cuestión de la escuela socialista", de Nadezhda Krupskaya, publicado por primera vez en 1918. Cita traducida del portugués al español por la autora. 
Internacional. En esta andadura estuvo acompañada por Rosa Luxemburgo en muchas ocasiones, a la que conoció en el año 1898, y con la que entabló una fuerte amistad y militancia común.

Las demandas más frecuentes de Zetkin se centraban en los derechos de las trabajadoras, poniendo de manifiesto sus problemas y la escasa participación política permitida a las mujeres -en Alemania, las mujeres no pudieron intervenir en ninguna actividad política hasta el año 1908-. Además, se le adjudica el papel de haber impulsado, dentro del Congreso de la Segunda Internacional en Copenhague de 1910, la propuesta de celebrar el 8 de marzo como el Día Internacional de la Mujer, honrando a las 129 mujeres que perdieron la vida en 1909 cuando protestaban para conseguir mejores derechos laborales en una fábrica textil en la ciudad de Nueva York. Además de su feroz lucha contra el imperialismo, y posteriormente contra el nazismo que surgiría en su propio país, Zetkin también escribió sobre la importancia de la

Imagen 2. Clara Zetkin y Nadezhda Krupskaya, 1927.

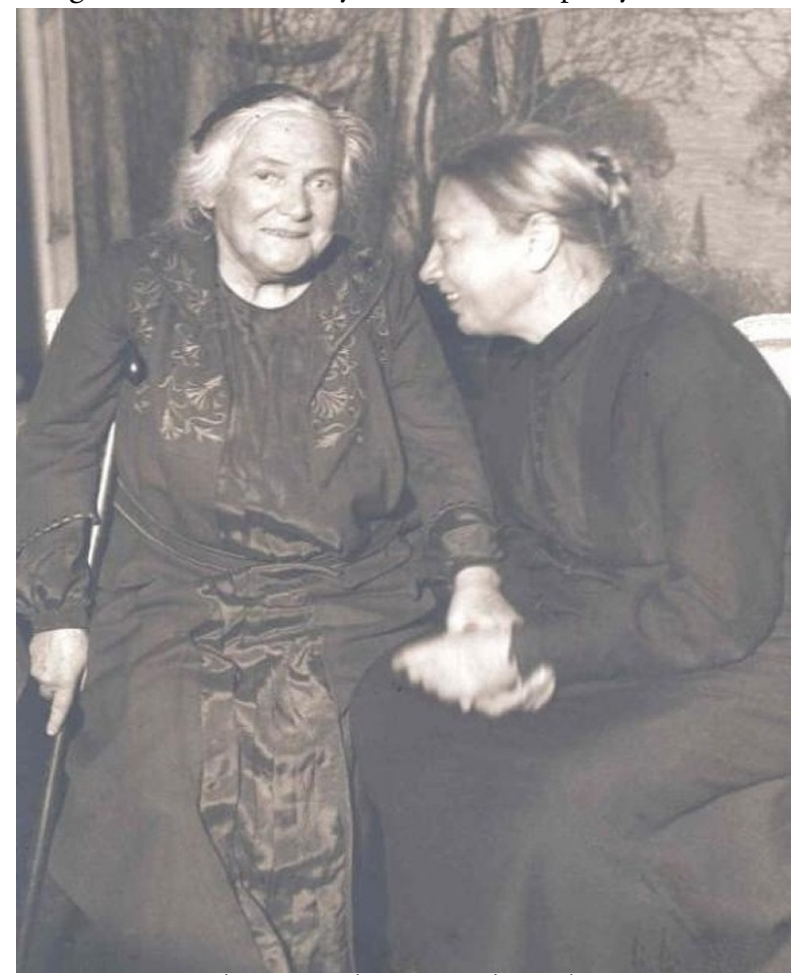

Fuente: Autor desconocido. Via Wikimedia Commons. 
participación política de las mujeres y propuso que existieran delegadas que pudieran asumir la dirigencia, así como defender los derechos específicamente de las mujeres.

Zetkin conoce a Lenin antes de la Revolución de Octubre, pero no visita Rusia hasta el año 1920, país en el cual decide fijar su residencia en diferentes momentos de su vida hasta su muerte. Desarrolló una amplia producción escrita y es la autora más citada por Krupskaya en sus escritos. Krupskaya define a Zetkin como "una de las líderes más destacadas y talentosas del movimiento de mujeres obreras en Alemania" (Krupskaya, 2017: 27) ${ }^{16}$. Asimismo, en otro artículo, "Acerca de la autocapacitación", publicado en 1935, Krupskaya vuelve a citar profusamente a Zetkin, al hacer referencia a sus palabras pronunciadas en diversos congresos en 1895 y en 1908.

Krupskaya no solo cita a Zetkin, sino que comparten también demandas similares, especialmente en relación con la participación de las mujeres en la política. En este caso, transcurrida ya una década tras la Revolución rusa, Krupskaya, en "Las obreras y campesinas en los Consejos", un artículo publicado en 1927, muestra su preocupación hacia la todavía escasa participación de las mujeres obreras y campesinas en dichos organismos. Consigue ofrecer datos concretos acerca de la intervención de las mujeres en los Consejos, que demuestran que continuaba siendo muy esporádica. A través de datos, muestra cómo, en las zonas rurales de la Federación Rusa en 1922, la participación de las mujeres apenas alcanzaba el $1 \%$, y que este porcentaje solo aumentó al $11,8 \%$ en el año 1927. En las zonas urbanas, en 1922 el porcentaje era del $9,8 \%$, y se incrementó hasta un $21,4 \%$ en 1927 . Como señala Krupskaya, un aumento de 10 puntos en 10 años no era suficiente (ídem: 145-146). Según ella, esa preocupante escasa participación de las mujeres era debida a las injerencias de los hombres del Consejo, que en muchas ocasiones no permitían a las mujeres formar parte del mismo; o a las dificultades que ellas mismas tenían para poder acceder a las reuniones por el exceso de trabajo en el hogar y la falta de apoyo por parte de sus maridos. A partir de las palabras de las mujeres campesinas, Krupskaya relata:

Ellas hablan sobre la "falta de conciencia" de los hombres, que miran con desdén a las mujeres miembros del Consejo de aldeas y no las ayudan de forma alguna [...]. Ellas se quejan de que sus maridos no tienen conciencia, de que

${ }^{16}$ Cita traducida del portugués al español por la autora. 
tienen que pelear con ellos porque para ellos nosotras "no tenemos que sufrir trabajando en los Consejos". Las campesinas dicen que las obreras están más acostumbradas a vivir en sociedad, están más unidas que ellas, y las campesinas están más aisladas, y por eso, los hombres se burlan de ellas [...]. Las obreras y las campesinas hablan mucho de su trabajo en el Consejo. Ellas dicen también que es necesario tener más círculos sobre el trabajo en el campo, cursos y entrenamiento (ídem: $147-148)^{17}$.

Otro ejemplo se muestra en el discurso pronunciado por Krupskaya en el VIII Congreso del Komsomol en el año 1928, donde pone de manifiesto que la discriminación hacia las mujeres también existía en los Komsomoles. Como el Komsomol "sabe -los manuales políticos lo dicen-, que, en nuestro país, las mujeres tienen los mismos derechos que los hombres, pero no nota en la vida que su hermanita no va a la escuela. Habla a menudo del kulak explotador, pero, a veces, no ve los efectos de la explotación donde los hay" (Krupskaya, 1978: 39). O cómo había niñas de 9 a 10 años de edad que eran traídas a la ciudad desde la aldea, huérfanas o semihuérfanas, para cuidar a los niños de las familias que trabajaban en los Comisariados, sin que esos trabajadores se diesen cuenta de que existía una explotación en esa acción y que esas niñas deberían estar asistiendo a la escuela (ibídem). A pesar de formar parte del Gobierno soviético, Krupskaya reconocía que todavía faltaba mucho para conseguir cambiar la situación de desigualdad de las mujeres trabajadoras rusas.

\section{La transformación de la familia: Krupskaya y Alexandra Kollontai}

A pesar de los desafíos que todavía existían para las mujeres después de la Revolución rusa, Krupskaya sí reconoce que algunas cosas habían cambiado, y pone como ejemplo a la familia. Esta institución, antes de la Revolución, podía llegar a suponer una condición de esclavitud para las mujeres, y esa situación se superó con las leyes que instauró el Derecho soviético, que contemplaban, entre otras, la posibilidad de solicitar el divorcio. Krupskaya se muestra crítica con la institución burguesa del matrimonio y considera que debe ser transformada, pero no llega a incluir en sus luchas la liberación sexual de las mujeres. Este era un tema controvertido en esa época, con autoras como

\footnotetext{
${ }^{17}$ Fragmento del texto "Las obreras y las campesinas en los consejos", de Nadezhda Krupskaya, publicado por primera vez en 1927. Cita traducida del portugués al español por la autora. 
Zetkin, que consideraban que el papel de las mujeres dentro del socialismo debía ser el de madres o el de esposas, sin llegar a transformar la estructura de la familia. Contrariamente a esta postura, Kollontai fue una de las pioneras a la hora de escribir sobre la revolución sexual de las mujeres unida a la lucha de la clase obrera.

Alexandra Kollontai, 1872-1952, nacida en San Petersburgo en una familia de la aristocracia, mostró desde una edad muy joven un ímpetu rebelde. Estudió a pesar de que su familia no se lo permitiera, se casó en contra de la voluntad de su familia y se divorció al poco tiempo, algo que no se consideraba adecuado en la época. Desde 1899 se afilió al Partido Obrero Social Democrático Ruso y decidió ir a Zúrich a estudiar, dejando a su hijo al cargo del que sería su primer marido, Vladímir Lúdvigovich Kollontai, del que más tarde acabaría divorciándose. Desde Zúrich, “Alejandra anduvo en busca de las teóricas feministas y, en particular, de Clara Zetkin y Rosa Luxemburgo. Al regresar en 1917, ya era amiga y colaboradora de Nadezhda Krúpskaya e Inessa Armand y formó parte con ellas del primer gobierno bolchevique, creado tras la Revolución de Octubre" (Gargallo, 2018: 20). El trabajo en unión con Krupskaya fue fundamentalmente dentro del Jenotdel, que Kollontai ayudó a crear y posteriormente dirigió al fallecer Armand; al igual que trabajó en el periódico Kommunistka. Pero, como hemos señalado, el vínculo no fue solo con las mujeres soviéticas, sino también con las alemanas, especialmente con Zetkin, al acudir en 1907 a la Primera Conferencia de las Mujeres Socialistas que esta organizaba, y al viajar y militar en el Partido Socialdemócrata con Rosa Luxemburgo en Alemania (ídem: 22-23).

A partir de la Revolución de Octubre en 1917, Kollontai formó parte del Comité Central del Partido, y ostentó el cargo de Comisaria del Pueblo para la Seguridad Social, con lo que se convirtió en la primera mujer en desempeñar un puesto de dirigencia dentro del Gobierno soviético. Algunas de sus preocupaciones, que compartía con sus compañeras feministas, fueron la erradicación de la prostitución, la relevancia de la lucha obrera de las mujeres -en oposición al movimiento feminista burgués-, y -al igual que Krupskaya-, la importancia de la instrucción de las mujeres, que estaba profundamente vinculada con la ruptura del yugo doméstico. Kollontai, además, defendía el derecho a las mujeres trabajadoras a solicitar el divorcio y a no depender económicamente de sus esposos: "En virtud del decreto del Comisario del Pueblo del 18 de diciembre de 1917, el divorcio ha dejado de ser un lujo 

socialistas en la Revolución rusa...

accesible solo a los ricos; desde ahora en adelante, la mujer trabajadora no tendrá que esperar meses, e incluso hasta años, para que sea fallada su petición de separación matrimonial que le dé derecho a independizarse de un marido borracho o brutal" (Kollontai, 2018: 75) ${ }^{18}$. Kollontai luchó arduamente para que las cuestiones de la opresión de las mujeres y su participación en la vida política se integraran en los debates del Partido Comunista (Bryant, 1923).

Imagen 3. Alexandra Kollontai (a la derecha) y Clara Zetkin (a la izquierda), 1921.

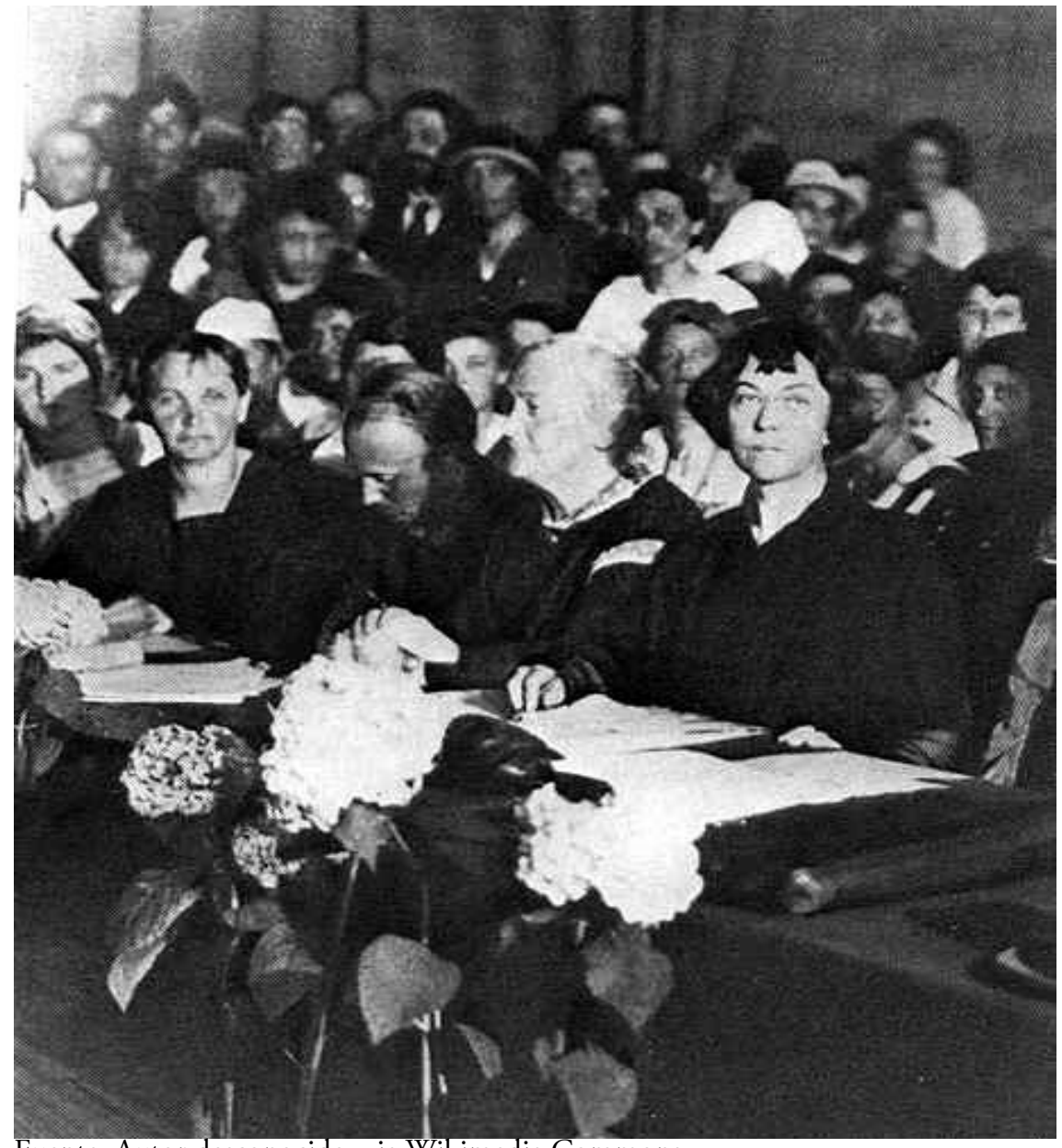

Fuente: Autor desconocido, via Wikimedia Commons.

\footnotetext{
${ }^{18}$ Fragmento del texto "El comunismo y la familia", de Alexandra Kollontai, publicado por primera vez en 1918. 
Ante el inicio de la burocratización del Estado Soviético, Kollontai formó parte, en 1921, de la Oposición Obrera, una corriente política que surgió cuando un grupo del Partido se opuso a la firma de la Paz de BrestLitovsk, por considerar que era un momento importante para impulsar una revolución socialista en otros países como Alemania, Bulgaria o Turquía. Este enfrentamiento hizo que Kollontai renunciara a la Comisaría del Pueblo y al Comité Central del partido (Gargallo, 2018). A partir de 1922, la Oposición Obrera se desintegró y Kollontai ya no volvió a sumarse a los debates del Partido Comunista Ruso. Además, coincide que en ese mismo año asume el puesto como embajadora, primero en Noruega, luego en México y finalmente en Suecia, convirtiéndose así en la primera mujer del mundo en ser embajadora.

Kollontai expresaba en sus textos cómo la emancipación femenina solo sería posible si a la par se producía una transformación en la sociedad, a nivel socioeconómico con leyes que regularan el trabajo y leyes que protegieran la maternidad -que contemplaran derechos como el descanso obligatorio para las embarazadas o las visitas médicas regladas-, al igual que jardines de infancia y espacios para embarazadas y recién nacidos, algo que impulsó dentro de las medidas adoptadas por el nuevo Gobierno soviético ${ }^{19}$. Krupskaya compartía con Kollontai la importancia de defender los derechos de las mujeres embarazadas, prácticamente inexistentes antes de la Revolución rusa. En "La mujer y la educación de la infancia", un artículo publicado en 1901 en la revista Iskra, Krupskaya denuncia que en la legislación de ese momento no se preveían derechos para las mujeres embarazadas, ya que el hecho de proporcionarles algún tipo de prestación dependía enteramente de que el patrón decidiera otorgarlo y, al no ser obligatorio, en pocas ocasiones se les concedía la ayuda. "La verdad, esos beneficios casi nunca son concedidos. Sin recibir los beneficios y con miedo a perder el trabajo, las mujeres trabajan en la fábrica casi hasta el último día del embarazo e intentan volver al trabajo lo antes posible, incluso sin estar recuperadas del parto. Por eso son tan frecuentes los abortos, los partos prematuros y todos los tipos de enfermedades ginecológicas en la fábrica” (Krupskaya, 2017: 25) ${ }^{20}$.

\footnotetext{
19 "Alexandra Kollontai introdujo dieciséis semanas de permiso de maternidad, el derecho al aborto, los derechos de las madres lactantes a no trabajar más de cuatro días a la semana y tiempo libre regulado para dar el pecho. [...] Se instituyó que todas las mujeres, sin importar si estaban casadas o no, recibieran su salario aunque se ausentasen del trabajo si iban a ayudar a amigas y compañeras laborales o políticas con el nacimiento de sus hijas e hijos" (Gargallo, 2018: 25).

${ }^{20}$ Cita traducida del portugués al español por la autora.
} 
En relación con la familia y el matrimonio, Kollontai tenía expectativas de un cambio en el futuro; "una nueva forma de familia que supondrá relaciones completamente diferentes entre el hombre y la mujer, basadas en una unión de afectos y camaradería, en una unión de dos personas iguales en la Sociedad comunista, las dos libres, las dos independientes, las dos obreras" (Kollontai, 2018: 98) ${ }^{21}$. En sintonía con estas pretensiones, Krupskaya señala que antiguamente "la vida familiar se basaba en la esclavitud: los hijos eran propiedad de los padres, y la mujer, del marido", y cómo "bajo el capitalismo se debilita la forma impositiva del matrimonio, pero este no deja de ser una operación comercial” (Krupskaya, 1978: 49-50). Krupskaya coincide con Kollontai en la necesidad de cambiar el matrimonio hacia una unión más libre y de amor mutuo. "Marx y Engels escribían ya que solo en el seno del proletariado podían surgir nuevas formas de relaciones matrimoniales: el matrimonio basado en la mutua simpatía, en el mutuo amor, en la mutua confianza y en la identidad de opiniones" (ídem: 50) ${ }^{22}$.

\section{Silenciadas pero no olvidadas}

A pesar de la relevancia de la producción escrita y el alcance de las propuestas y acciones de las mujeres en la Revolución rusa, está poco valorado en la actualidad el papel fundamental que tuvieron dentro del proceso revolucionario ruso. Graziela Schneider (2017) nos muestra la gran diversidad de mujeres importantes durante ese período en Rusia. Entre ellas, destacan Anna Andréievna Kalmánovitch -activista del movimiento de mujeres y una de las primeras en impartir conferencias sobre los derechos de las mujeres-; Olga Andréievna Chapir - de origen campesino, profesora de universidad y miembro de la Unión por la Igualdad de los Derechos de la Mujer-; María Ivánva Pokróvskaia -doctora, editora de la revista Jénski Vêstnik y fundadora del Partido Progresista de las Mujeres-; Liubov Iákovlevna Guriévitch editora, escritora, periodista, y militante de organizaciones políticas de mujeres en Rusia-; Ekaterina Dmítrievna Kuskova -editora de varias revistas y miembro de la Liga para la Igualdad de Derechos de la Mujer-; o Konkórdia Nikoláievna Samóilova -revolucionaria, activista, fundadora del periódico Pravda, miembro editorial de Rabotnitsa y organizadora junto con Kollontai

\footnotetext{
${ }^{21}$ Fragmento del texto "El comunismo y la familia", de Alexandra Kollontai, publicado por primera vez en 1918.

${ }^{22}$ Fragmento del texto "Importante sector del trabajo del Komsomol", de Nadezhda Krupskaya, publicado por primera vez en 1935 .
} 
del Primer Congreso de Trabajadoras de Toda Rusia-. No es posible mostrar en este trabajo la extensa trayectoria de todas estas mujeres, teniendo en cuenta además que asumieron diferentes posturas políticas que influyeron en sus perspectivas en relación con el feminismo. Pero sí es necesario señalar que todas ellas forman parte central de la historia de la Revolución rusa, donde asumieron puestos políticos y de militancia activa, escribieron en numerosas publicaciones, editaron periódicos, crearon partidos políticos y llevaron a cabo un significativo trabajo para defender y mejorar las condiciones de vida de las mujeres.

Pero aunque silenciadas a partir de 1930 en Rusia con el cierre del Jenotdel, estas autoras no han sido olvidadas en la actualidad. Además del impacto que tuvieron en la Revolución rusa y en el movimiento por los derechos de las mujeres de principios del siglo XX, es imprescindible señalar que las reivindicaciones que realizaban en ese momento todavía mantienen su vigencia en la actualidad. Por ejemplo, resulta significativo que una de las acciones del Jenotdel en los inicios de la Rusia Soviética fuera el derecho al aborto por decisión de las mujeres y gratuito, lo que constituye todo un hito histórico porque fue el primer país del mundo en legalizar el aborto. El trabajo del Jenotdel y de las mujeres que lucharon por conseguir este logro dialoga todavía hoy con una de las actuales demandas del feminismo, y muy recientemente, con mayor impulso en América Latina a través del movimiento de la "marea verde" surgido en Argentina ${ }^{23}$.

Otro de los grandes debates, la importancia de la defensa de los derechos laborales de las mujeres trabajadoras -algo que las autoras rusas reclamaban al describir cómo en las fábricas de su época las mujeres tenían una escasa libertad para defenderse de abusos o poder disponer de derechos por maternidad-, constituye todavía una de las luchas permanentes del feminismo frente a una violenta y feroz explotación laboral de las mujeres.

En cuanto a la ruptura del "yugo doméstico" que Krupskaya y Kollontai ${ }^{24}$ expresaban narrando la injusticia del doble trabajo que muchas mujeres realizaban en la fábrica y luego en el hogar, esta continúa siendo en la actualidad

\footnotetext{
${ }^{23}$ Movimiento a favor del derecho al aborto legal, seguro y gratuito en Argentina, que usa como símbolo los pańuelos verdes y el lema "Educación sexual para decidir, anticonceptivos para no abortar, aborto legal para no morir".

${ }^{24}$ En relación con las tareas del hogar, Kollontai afirma que "estos son trabajos agotadores. Consumen todas las energías y todo el tiempo de la mujer trabajadora, que, además, tiene que trabajar en una fábrica” (Kollontai, 2018: 87).
} 
una de las principales reivindicaciones del feminismo. Como, por ejemplo, en las marchas por el Día Internacional de la Mujer del 8 de marzo en Uruguay y en Argentina, donde, al igual que en otros países, una de las principales consignas fue "Eso que llaman amor es trabajo no pago", en referencia a las labores de cuidados que las mujeres desempeñan y que no son ni reconocidas ni remuneradas.

Un ejemplo específico de la incidencia de estas autoras se puede observar en el Movimiento de los Trabajadores Rurales Sin Tierra -MST- en Brasil. Desde su inicio, en el MST se hizo un fuerte hincapié en la participación política de las mujeres dentro de todos los niveles organizativos -local, estatal y nacional-, y en todos sus ámbitos de acción. Eligen siempre a un hombre y una mujer como coordinador y coordinadora, respectivamente, durante un tiempo determinado y, al ser relegados por otros, se mantiene siempre esa equidad de género. Pero, además, el MST tiene un Sector de Género que, desde una perspectiva feminista campesina popular, lleva a cabo diferentes actividades tanto de organización y formación como de concientización y acción política. Este Sector recoge, en los documentos que produce, múltiples referencias a autoras como Luxemburgo, Krupskaya, Zetkin y Kollontai, y reconoce su papel dentro del feminismo de la clase trabajadora, con el cual se identifica. Sin embargo, aunque admite la importante influencia que estas autoras han ejercido sobre el MST, señala que "también es preciso reconocer que el Feminismo Revolucionario, al cual nos vinculamos, quedó restringido por mucho tiempo a una perspectiva eurocéntrica, sin tener en consideración las realidades de las mujeres en otros países y regiones, sus culturas, sus modos de vida e interpretación de la realidad" (Oliveira Mafort, 2017: 202)25. Así pues, retoman como base de su pensamiento ese primigenio feminismo socialista europeo, para articular después, desde su especial contexto de lucha por una Reforma Agraria Popular en el campo brasileño, su propia teoría y práctica de un feminismo campesino popular y anticapitalista. Esta postura es, además, compartida con las compañeras de la Coordinadora Latinoamericana de Organizaciones del Campo -CLOC- y de la Vía Campesina.

Entre las numerosas iniciativas del Sector de Género y del Sector de Educación -de manera similar a lo que ocurría con las demandas de Krupskaya y de Armand de la necesaria implementación de jardines de infancia para los hijos e hijas de las mujeres trabajadoras-, el MST ha creado guarderías

${ }^{25}$ Cita traducida del portugués al español por la autora. 
infantiles para poder así permitir y facilitar la participación de las mujeres en todo tipo de eventos formativos, políticos, de trabajo productivo o de organización social. Estas guarderías infantiles, que se denominan Cirandas infantis, son espacios donde las madres y los padres militantes del MST pueden dejar a sus hijos e hijas al cuidado del colectivo de educación. De esta manera, pueden formar parte activa de las reuniones locales, regionales o nacionales del MST, y acudir a los distintos centros educativos donde se imparten cursos, formación al profesorado o instrucción política. Las Cirandas infantis son organizadas por el propio movimiento, y por tanto tienen también un fin político pedagógico, de instrucción de los hijos e hijas de los militantes del MST, a los que llaman Sem-Terrinhas.

Todos estos ejemplos nos permiten reflexionar acerca de los posibles diálogos de las ideas y logros de las mujeres socialistas rusas con los movimientos sociales actuales. Tanto aquellos con demandas feministas particulares -como el aborto libre, gratuito y seguro; la defensa de los derechos de las mujeres trabajadoras; la brecha de género en los trabajos de cuidados y del hogar, etc.como también los movimientos sociales que orientan sus objetivos políticos hacia otros ámbitos, pero que abogan por introducir el feminismo dentro de su forma de organización social, como es el caso del MST.

\section{Reflexiones finales}

Un aspecto llamativo de las mujeres socialistas que hemos presentado en este artículo es que, mientras que son defensoras de las mujeres como sujetos políticos y reivindican la existencia de una doble explotación de las mujeres, al mismo tiempo se alejan de lo que en esa época las feministas burguesas denominaban "la cuestión de la mujer". Krupskaya, en el artículo editorial del primer número de Rabotnitsa, publicado en 1914, establece lo siguiente:

Las mujeres burguesas defienden "sus derechos de las mujeres" especiales, siempre se oponen a los hombres y demandan sus derechos de los hombres. Para ellas, la sociedad contemporánea se divide en dos categorías principales, hombres y mujeres. Los hombres poseen todo, tienen todos los derechos. La cuestión es lograr la igualdad de derechos.

Para la mujer trabajadora, la cuestión de la mujer es muy diferente. Las mujeres políticamente conscientes ven que la sociedad contemporánea está dividida en clases. La burguesía es una clase, la clase trabajadora es otra. Sus intereses 
son contrapuestos. [...] La "cuestión de la mujer", para los trabajadores y las trabajadoras, consiste en cómo involucrar a las masas atrasadas de las mujeres trabajadoras en la organización, cómo dejarles en claro sus intereses, cómo hacer que se transformen rápidamente en compañeras de la lucha común (Krupskaya, citada en Frencia y Gaido, 2018: 61-62).

Para estas autoras, la defensa de las mujeres tiene como base la diferencia de clases, y a partir de ese posicionamiento político es desde donde proyectan su lucha. Como vimos al inicio del artículo, la relación entre el feminismo y el socialismo ha sido compleja. Hartmann (1979) advierte cómo muchos marxistas han establecido el feminismo como un objetivo secundario, como si las demandas de las mujeres se fueran a obtener tan solo por transformar las relaciones económicas y sociales del capitalismo. "Si bien el análisis marxista aporta una visión esencial de las leyes del desarrollo histórico, y de las del capital en particular, las categorías del marxismo son ciegas al sexo" (Hartmann, 1979: 2). Pero Hartmann no descarta el marxismo, al contrario, lo considera necesario, complementándolo con una perspectiva feminista. "Hay que recurrir tanto al análisis marxista, y en particular a su método histórico y materialista, como al análisis feminista, y en especial a la identificación del patriarcado como estructura social e histórica, si se quiere entender el desarrollo de las sociedades capitalistas occidentales y la difícil situación de la mujer dentro de ellas" (ibídem).

Para este fin, el de entablar el diálogo entre el feminismo y el marxismo, las autoras socialistas rusas nos pueden resultar de gran ayuda. Krupskaya, a pesar de creer firmemente en la lucha obrera como objetivo político, tampoco deja de lado en sus textos una mirada crítica hacia las actitudes patriarcales en el seno del Gobierno soviético o en los Komsomoles. Sin llegar a establecer que el problema reside en el patriarcado, sí llega a denunciar el trato desigual e injusto hacia las mujeres, en especial en el acceso a la política y a la educación (Krupskaya, 1978). Kollontai denuncia la visión individualista burguesa que creía que los asuntos concernientes a la sexualidad debían ser algo privado e íntimo, pero al mismo tiempo critica a los socialistas que opinaban que las relaciones entre hombres y mujeres eran aspectos secundarios, solucionables a futuro cuando la sociedad capitalista dejara de existir. "La solución de los problemas sexuales solo podrá hallarse en el establecimiento de un orden social y económico nuevo, con una transformación fundamental de nuestra sociedad 
actual', afirman los socialistas. Pero, precisamente este esperar en el mañana, ¿no indica también que nosotros tampoco hemos logrado apoderarnos del hilo conductor'?" (Kollontai, 2018: 48) ${ }^{26}$.

A pesar de la oposición ideológica al feminismo de esa época, las acciones y logros de las mujeres socialistas rusas han marcado verdaderos hitos en la historia de las mujeres, y por ende forman parte de la lucha histórica del feminismo. El período comprendido entre 1917 hasta la década de los años 30 vio florecer grandes cambios en relación con los derechos de las mujeres en Rusia: el divorcio se facilitó y se concedía automáticamente, se estableció que los hijos e hijas dentro o fuera del matrimonio tuvieran los mismos derechos, se garantizó el permiso de maternidad, se concedieron más derechos a las mujeres en el trabajo y se estableció la igualdad de los cónyuges en relación con los hijos e hijas (Navailh, 1993: 259). Además, las acciones de las mujeres socialistas rusas no solo se circunscriben a las políticas promovidas una vez instaurado el Gobierno soviético. Desde antes de la Revolución de 1917, más mujeres que hombres fueron deportadas a Siberia por organizar revueltas en contra del zar (Bryant, 1923), lo que denota su amplia participación en los movimientos prerrevolucionarios.

No es usual el reconocimiento explícito de que el inicio de la Revolución de Febrero de 1917 tuvo lugar en San Petersburgo, cuando, durante la celebración del Día Internacional de la Mujer -el 8 de marzo equivale al 23 de febrero en el calendario ruso-, se manifestaron masivamente las obreras textiles demandando pan y paz. Así lo reivindica Kollontai, con claridad y rotundidad, en referencia al Día de la Mujer Trabajadora:

Pero este no es un día especial solo para las mujeres. El 8 de marzo es un día histórico y memorable para los obreros y campesinos, para todos los trabajadores rusos y para los trabajadores de todo el mundo. En 1917, en este día, estalló la gran Revolución de Febrero. Fueron las mujeres obreras de San Petersburgo quienes comenzaron esta revolución; fueron ellas las primeras en levantar la bandera de oposición al Zar y sus compinches. Y así, para nosotras, el día de la mujer trabajadora es un día de doble celebración (Kollontai, 1920).

Con esta referencia al Día de la Mujer Trabajadora de 1917, se considera necesario destacar la trascendencia del 8 de marzo en la actualidad, y cómo

${ }^{26}$ Fragmento del texto "Las relaciones sexuales y la lucha de clases", de Alexandra Kollontai, publicado por primera vez en 1911. 
los pasados tres años, desde 2017 hasta ahora, han sido testigos de una extraordinaria huelga feminista que ha tomado las calles reuniendo el mismo día a millones de mujeres en diferentes países. A partir de esa reflexión quisiéramos reivindicar el papel de estas mujeres socialistas rusas, por su poderoso legado, y por la interlocución que ellas y sus ideas todavía establecen con los desafíos que se nos presentan en la actualidad.

\section{Bibliografía citada}

Armand, Inessa, (1920) "La obrera en la Rusia Soviética", en Bulletin Communiste. 17, pp.12-15. Recuperado de: https://www.marxists. org/espanol/armand/la-obrera-sovietica.pdf [fecha de consulta: 17 de marzo de 2019].

Bryant, Louise, (1923) Mirrors of Moscow. New York: T. Seltzer (ed.). Recuperado de: https://www.marxists.org/archive/bryant/ works/1923-mom/mirrors-full.htm [fecha de consulta: 17 de marzo de 2019].

Capel Martínez, Rosa Ma , (2008) “Mujer y socialismo (1848-1939)”, en Pasado y Memoria. Revista de Historia Contemporánea. 7, pp. 101-122. De Miguel Álvarez, Ana, Eva Palomo Cermeño, (2018) “De las revueltas de 'pan y paz' a la política de organización y comunicación: las mujeres en la revolución rusa”, en Historia y comunicación social. 23, 1, pp.3348.

Elwood, Ralph Carter, (1992) Inessa Armand. Revolutionary and Feminist. New York: Cambridge University Press.

Felis, Clara, (2017) "Las tres mujeres que utilizó Lenin para el triunfo de la Revolución Rusa”, en Periódico el Mundo. 8 de marzo del 2017.

Frencia, Cintia y Daniel Gaido, (2018) Feminismo y movimiento de mujeres socialistas en la Revolución Rusa. Santiago de Chile: Ariadna ediciones.

Gargallo, Francesca, (2018) "Presentación. Alejandra Kollontai: amor libre y libertad social. La vigencia de una necesidad", en Alejandra Kollontai, Alejandra Kollontai: El comunismo y la familia. Las relaciones sexuales y la lucha de clases. Bogotá: Ediciones desde abajo, pp. 7-44. 

socialistas en la Revolución rusa...

Haigh, Maria, (2007) “Escaping Lenin's Library: Library and Information Science Education in Independent Ukraine", en The International Information \&Library Review. 39, 2, pp. 72-79.

Hartmann, Heidi, (1979) "Un matrimonio mal avenido: hacia una unión más progresiva entre marxismo y feminismo", en Papers de la Fundació, 88. Fundació Rafael Campalans. (en línea). Recuperado de: https:// fcampalans.cat/archivos/papers/88.pdf.

Hernández Navarro, Luis, (2017) "La educación bolchevique, al cielo por asalto", en La Jornada. 17 de Octubre del 2017.

Kollontai, Alejandra, (2018) Alejandra Kollontai: El comunismo y la familia. Las relaciones sexuales y la lucha de clases. Bogotá: Ediciones desde abajo.

Kollontai, Alejandra, (2017), [1913] "A mulher trabalhadora na sociedade contemporânea”, en Graziela Schneider (org.), A Revolução das mulheres: emancipação femenina na Rússia soviética. São Paulo: Boitempo, pp. 150-159.

Kollontai, Alejandra, (1920) El Día Internacional de la Mujer. (en línea). Recuperado de: https://www.marxists.org/espanol/ kollontai/1920/0001.htm [fecha de consulta: 17 de marzo del 2019].

Krupskaya, Nadezhda, (2017) A construção da pedagogía socialista (Escritos seleccionados). São Paulo: Expressão Popular.

Krupskaya, Nadezhda, (1978) La educación de la juventud. Madrid: Nuestra Cultura. (en línea). Recuperado de: http://bolchetvo.blogspot. $\mathrm{mx} / 2011 / 04 /$ la-educacion-comunista.html [fecha de consulta: 17 de marzo del 2019].

Muñoz-Muñoz, Ana María, (2010) "Nadezhda Konstantinovna Krupskaia (1869-1939): Feminista y Bibliotecaria”, en Ana María MuñozMuñoz y Pilar Ballarín Domingo (eds.), Mujeres y libros. Homenaje a la profesora Dña. Isabel de Torres Ramírez. Granada: Universidad de Granada. pp. 143-156.

Narodnaya Volya: "La Voluntad del Pueblo", (2015) Revista Nada. (en línea). Recuperado de: https://revistanada.com/2015/03/28/narodnayavolya-la-voluntad-del-pueblo/ [fecha de consulta: 17 de marzo del 2019].

Nash, Mary, (2004) Mujeres en el mundo. Historia, retos y movimientos. Madrid: Alianza Editorial. 
Navailh, Françoise, (1993) “El modelo soviético", en Georges Duby y Michelle Perrot (direc.), Historia de las mujeres en Occidente. 9. México-España: Taurus. pp. 257-283.

Oliveira Mafort, Kelli Cristine de, (2017) "A construção de novas relações sociais de gênero no MST e a luta pela emancipação humana", en Roseli Salete Caldart y Rafael Litvin Villas Bôas (orgs.), Pedagogia Socialista. Legado da revolução de 1917 e desafíos atuais. São Paulo: Expressão Popular.

Reich, Wilhelm, (1985) La revolución sexual: para una estructura de carácter autónoma del hombre. Barcelona-México: Planeta De AgostiniEditorial Artemisa.

Sargent, Lydia y Heidi Hartmann, (1981) The Unhappy Marriage of Marxism and Feminism. A Debate of Class and Patriarchy. Londres: Pluto.

Schneider, Graziela (org.), (2017) A revolução das mulheres: emancipação feminina na Rússia soviética. Artigos, atas, panfletos, ensaios. São Paulo: Boitempo.

Skatkin, Mihail, Georgij Cov'janov, (1994) "Nadezhda Krupskaya (18691939)", en Prospects: the Quarterly Review of Comparative Education. 24, 1/2, pp. 49-60. Recuperado de: http://www.ibe.unesco.org/sites/ default/files/krupskae.pdf [fecha de consulta: 17 de marzo del 2019]. Trotsky, Leon, (1939) “Krupskaya's Death", en The New International. 5. 4. pp.117. Recuperado de: https://www.marxists.org/archive/ trotsky/1939/03/krupskaya.htm [fecha de consulta: 17 de marzo del 2019].

Yegorov, Oleg, (2017) "El «revolucionario» triángulo amoroso de Lenin", en Russia Beyond. 26 de mayo del 2017.

\section{Fuente de las imágenes}

Alexandra Kollontai y Clara Zetkin, (1921) Autor desconocido, via Wikimedia Commons. Recuperado de: https://commons.wikimedia.org/wiki/ File:Zetkin_Kollontaj_Comintern.jpg

Clara Zetkin y Nadezhda Krupskaya, (1927) Autor desconocido, via Wikimedia Commons. Recuperado de: https://commons.wikimedia. org/wiki/File:Clara_Zetkin_\%26_Nadeshda_Krupskaja_1927.jpg 

socialistas en la Revolución rusa...

Revista Rabotnitsa, (1923), Autor desconocido, via Wikimedia Commons. Recuperado de: https://upload.wikimedia.org/wikipedia/ commons/9/93/Rabotnitsa.jpg 